\title{
Textured semiconductors for enhanced photoconductive terahertz emission
}

\author{
Christopher M. Collier, Jeffrey D. A. Krupa, Ilija R. Hristovski*, Trevor J. Stirling, Mark H. Bergen, \\ and Jonathan F. Holzman \\ Integrated Optics Laboratory, The University of British Columbia, School of Engineering \\ 3333 University Way, Kelowna, BC, Canada, V1V 1V7
}

\begin{abstract}
There are severe limitations that photoconductive (PC) terahertz (THz) antennas experience due to Joule heating and ohmic losses, which cause premature device breakdown through thermal runaway. In response, this work introduces PC $\mathrm{THz}$ antennas utilizing textured InP semiconductors. These textured InP semiconductors exhibit high surface recombination properties and have shortened carrier lifetimes which limit residual photocurrents in the picoseconds following THz pulse emission-ultimately reducing Joule heating and ohmic losses. Fine- and coarse-textured InP semiconductors are studied and compared to a smooth-textured InP semiconductor, which provides a baseline. The surface area ratio (measuring roughness) of the smooth-, fine-, and coarse-textured InP semiconductors is resolved through a computational analysis of SEM images and found as $1.0 \pm 0.1,2.9 \pm 0.4$, and $4.3 \pm 0.6$, respectively. The carrier lifetimes of the smooth-, fine-, and coarse-textured InP semiconductors are found as respective values of $200 \pm 6$, $100 \pm 10$, and $20 \pm 3$ ps when measured with a pump-probe experimental system. The emitted THz electric fields and corresponding consumption of photocurrent are measured with a $\mathrm{THz}$ experimental setup. The temporal and spectral responses of PC THz antennas made with each of the textured InP semiconductors are found to be similar; however, the consumption of photocurrent (relating to Joule heating and ohmic losses) is greatly diminished for the semiconductors that are textured. The findings of this work can assist in engineering of small-scale PC THz antennas for high-power operation, where they are extremely vulnerable to premature device breakdown through thermal runaway.
\end{abstract}

Keywords: Pump-probe spectroscopy, terahertz generation, ultrafast photonics

\section{INTRODUCTION}

Since their emergence, mode-locked ultrafast oscillating lasers have enabled many pursuits in science, e.g., ultrafast material characterization studies [1,2], advancements in surgical instrumentation [3], and the detection and generation of radiation in the terahertz $(\mathrm{THz})$ portion of the electromagnetic spectrum [4]. The frequencies from $0.1-10 \mathrm{THz}$ (or wavelengths from 30-3,000 $\mu \mathrm{m}$ ) define the $\mathrm{THz}$ spectrum and have received considerable attention in past decades [5-9]. Technologies involving $\mathrm{THz}$ radiation have provided numerous advancements in applications of applied science, e.g., tomography [10], characterization of semiconductors [11, 12], security [13], and many biomedical applications, e.g., medical imaging [14], medical diagnosis [15], and analyses of DNA [16]. The development of the above applications in past years has run in parallel with advancements in $\mathrm{THz}$ detection and generation. Terahertz generation can occur as continuous-waves, by difference-frequency generation in electro-optic crystals [17] or by heterodyne mixing in semiconductors [18]. Terahertz generation can also occur as pulses, by optical rectification in electro-optic crystal emitters [19] or by exciting charge-carriers in photoconductive (PC) THz emitters with bias fields present [4].

Terahertz emitters with $\mathrm{THz}$ electric fields that are scalable with both bias voltage amplitudes and excitation optical fluences are particularly noteworthy, e.g., PC THz emitters [20]. In these PC THz emitters, THz pulses of electric field radiate due to charge-carrier acceleration in the picoseconds following excitation with an optical pulse. One downside of PC THz emitters is that the charge-carrier lifetime is typically much longer than the picosecond duration of the terahertz transient, which leads to residual photocurrents that cause ohmic losses and Joule heating. These effects impede the desired scaling up of the THz electric field through raising the bias voltage amplitude or excitation optical fluence [21]. (The thermal runaway onset and the excitation optical fluence have an inverse relationship [22].) At the same time, the $\mathrm{THz}$ electric field cannot be increased by raising the repetition frequency of the excitation laser because this would raise the Joule heating, which has a proportionality to the repetition frequency of incident excitation optical pulses [23].

Terahertz, RF, Millimeter, and Submillimeter-Wave Technology and Applications IX,

edited by Laurence P. Sadwick, Tianxin Yang, Proc. of SPIE Vol. 9747, 97470M

(C) 2016 SPIE · CCC code: $0277-786 X / 16 / \$ 18 \cdot$ doi: $10.1117 / 12.2208548$

Proc. of SPIE Vol. $974797470 \mathrm{M}-1$ 
Researchers have responded to the discussed challenges through investigations of PC THz emitters that use large active areas [24]; however, these devices still suffer from significant ohmic losses and Joule heating. Additionally, there are many applications that have considerable spatial constraints, e.g., on-chip biofluid systems [25-27]. For compatibility with such systems, PC THz emitters must have small active areas. These $\mathrm{THz}$ systems would be particularly susceptible to ohmic losses and Joule heating as the onset voltage for thermal runaway has an inverse relationship to the active area of the PC THz emitter [22].

Existing solutions to these problems are complex. These proposed solutions include cooling with water [28], irradiation [21], elaborate designs for bias electrodes [29], active area patterning [30], and heat sinks [31]. Given these cumbersome proposed solutions, this work focuses on a PC THz emitter that is based on a simple but effective material, textured InP. This texturing of the InP surface increases the surface area that is excited by the incident optical pulses, and therefore increases surface effects, leading to an overall decrease in the charge-carrier lifetimes [32,33]. The work on textured InP materials includes three investigations: there is a scanning electron microscope (SEM) analysis that quantifies surface roughness; there is a study of the ultrafast material response of smooth-, fine-, and coarse-textured InP materials with a pump-probe system that measures charge-carrier lifetime; and there is an investigation of the THz response of smooth-, fine-, and coarse-textured $\mathrm{InP}$ materials with a $\mathrm{THz}$ system that measures relative $\mathrm{THz}$ electric field strength and photocurrent consumption. Similar characteristics (temporal and spectral) are observed in emitters with varying texturizations, however, consumption of photocurrent is reduced greatly in $\mathrm{PC} \mathrm{THz} \mathrm{emitters} \mathrm{constructed} \mathrm{from} \mathrm{textured}$ materials.

\section{METHODS}

A pump-probe system is used to measure charge-carrier lifetimes of the InP materials. Figure 1(a) shows a top view of the pump-probe system, which uses an ultrafast pulsed laser with $780 \mathrm{~nm}$ (pump beam) and $1550 \mathrm{~nm}$ (probe beam) pulses with $100 \mathrm{fs}$ duration [34]. The $1.3 \mathrm{eV}$ InP bandgap resides between the photon energies $(1.6$ and $0.8 \mathrm{eV}$ respectively) of the pump and probe beams. The pump and probe beams are overlapped with a dichroic beamsplitter (labeled DBS) and then pass through a 40 times microscope objective (labeled $\mathrm{MO}_{40}$ ) onto a material sample (labeled MS). The position of the material sample can be adjusted as it is mounted on an xyz stage (labeled XYZS). As the pump pulses change the sample photoconductivity, the corresponding change in probe transmission is measured using an InGaAs photodiode (labeled IPD).

To quantify the InP PC emitter $\mathrm{THz}$ response, describing the relative $\mathrm{THz}$ electric field strength and consumption of photocurrent, a THz system can be used. Figure 1(b) shows a top view of the THz system, which uses an ultrafast pulsed laser with $780 \mathrm{~nm}$ pump and probe beams. The pump beam passes through a 10-times microscope object (labeled $\mathrm{MO}_{10}$ ) onto a PC THz emitter (labeled PTE) which is constructed from smooth-, fine-, and coarse-textured InP materials and silver epoxy electrodes [35-reid] with a $100 \mu \mathrm{m}$ gap spacing. The position of the PC THz emitter can be adjusted as it is mounted on an xyz stage (labeled XYZS). The THz radiation is collimated with a parabolic reflector (labeled PR) and is overlapped with the probe beam by means of a pellicle beamsplitter (PeBS). These collinear beams are then focused with another parabolic reflector into the ZnTe electro-optic crystal (labeled ZnTe) and through the quarter-wave plate (labeled $\lambda / 4$ ) and through the Wollaston prism (labeled WP) which divides the probe beam into its polarizations (vertical and horizontal). The difference of these polarizations (which is proportional to the THz electric field strength) is then measured with a set of differencing Si photodiodes (DSPD). At the same time, the photocurrent consumption in the PC $\mathrm{THz}$ emitter is measured with a transimpedance amplifier.

\section{RESULTS}

By increasing surface effects (through an increase in surface area), the charge-carrier lifetime of an optically-illuminated semiconductor can be reduced [32,33], which is a large advantage for PC THz emitters. To make textured InP materials, an InP material is mechanically polished using polishing films with different sizes of grit. Thorlabs LF6D $(6 \mu \mathrm{m})$ polishing paper and Thorlabs LF30D $(30 \mu \mathrm{m})$ polishing paper are used to produce fine- and coarse-textured InP materials, respectively. The relative surface area is defined as the unitless ratio of the textured sample surface area divided by the polished sample surface area, and this relative surface area quantifies the level of texturing on the InP material. The relative surface area can be measured for each sample through an analysis of images taken with a SEM. 
Here, many sites on each sample are included to ensure a representative result. An analysis of these images from the SEM is performed with a software topography program which converts the images to three dimensional maps from which surface areas are extracted. Figure 2 shows representative three dimensional images of the InP materials for (a) smooth-textured InP material, (b) fine-textured InP material, and (c) coarse-textured InP material. Based on ten different sites for each material, the smooth-, fine-, and coarse-textured $\mathrm{InP}$ materials are found to have respective relative surface areas of $1.0 \pm 0.1,2.9 \pm 0.4$, and $4.3 \pm 0.6$.

The THz electric field that radiates from a PC THz emitter follows the equation

$$
E_{T H z}(t) \propto \frac{d[\sigma(t)]}{d t} E_{b}=\frac{d[q \mu n(t)]}{d t} E_{b} .
$$

Here, the electric field is $E_{\mathrm{THz}}(t)$, the semiconductor conductivity is $\sigma(t)$, the elementary charge is $q$, the mobility of the semiconductor is $\mu$, the density of the charge-carriers is $n(t)$, and the electric field from the bias voltage is $E_{\mathrm{b}}$. Just after the pump beam excitation, there is a fast rise in conductivity that has a proportionality to the rapidly-rising charge-carrier density. This produces an electric field that is proportional to the time derivative of the conductivity. This conductivity remains high and gradually falls (over the material charge-carrier lifetime) with the density of the charge-carriers. The lasting conductivity leads to a residual photocurrent that is undesirable (as it produces Joule heating and ohmic losses). For this work, the InP mobility is assumed to have a constant value. However, it should be noted that other materials, e.g., GaP, have a transient mobility that can be used to limit residual photocurrents [36, 37].

In order to find the dissipated power, $P_{\text {dissipated }}$, from Joule heating and ohmic loss, the electrical power in the PC THz emitter can be integrated over one period of the ultrafast pulsed laser. This analysis yields a linearity between chargecarrier lifetime, $\tau$, and dissipated power through

$$
P_{\text {dissipated }}=\frac{K}{T_{0}} \int_{0}^{T_{0}} e^{-t / \tau} d t=\frac{K}{T_{0}} \tau\left(1-e^{-T_{0} / \tau}\right) \approx \tau \frac{K}{T_{0}} .
$$

Here, the period of the ultrafast pulsed laser is $T_{0}$ and the constant of proportionality is $K$. Equation (2) indicates that a short charge-carrier lifetime is desirable.

Using the aforementioned pump-probe system, the charge-carrier lifetimes of InP materials that are smooth-, fine-, and coarse-textured can be investigated. Figure 3 presents ultrafast differential transmission scans for each textured emitter

(a) pump-probe system

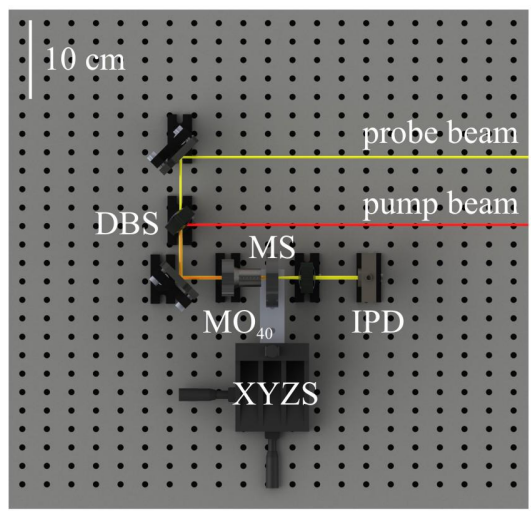

(b) terahertz system

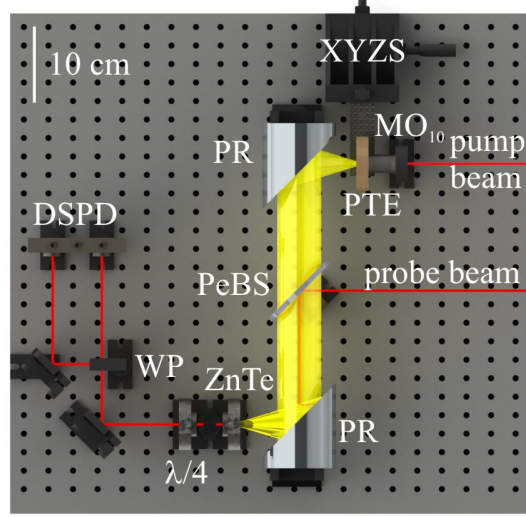

Figure 1. Schematics for (a) the pump-probe system and (b) the THz system. 
(smooth-, fine-, and coarse-textured InP materials are shown in solid lines coloured respectively in black, purple, and green). Here, differential transmission, $\Delta T / T$, versus time, $t$, is plotted. The scans decay according to exponential functions and curve fitting yields charge-carrier lifetimes of $200 \pm 6$ ps (smooth-textured InP), $100 \pm 10$ ps (fine-textured InP), and $20 \pm 3$ ps (coarse-textured InP). These lifetimes are taken as the mean of six scans with standard errors and approximately agree with InP literature values [38].

The $\mathrm{THz}$ response of $\mathrm{PC} \mathrm{THz}$ emitters based on each material can also be measured. This measurement is performed using the $\mathrm{THz}$ system. Here, the $\mathrm{THz}$ electric field that is radiated is measured along with the photocurrent that is consumed in the PC THz emitter. Each PC THz emitter produces approximately the same THz electric field at the same $50 \mathrm{~V}$ bias voltage. Figure $4(\mathrm{a})$ shows the normalized $\mathrm{THz}$ amplitude electric field, $E_{\mathrm{THz} \text {,norm }}\left(V_{\text {bias }}\right)$, versus the applied bias voltage, $V_{\text {bias}}$, shown as black triangles (smooth-textured InP), purple diamonds (fine-textured InP), and green squares (coarse-textured InP). The gap spacing of each emitter is made to be $100 \mu \mathrm{m}$. Figure 4(b) presents the corresponding photocurrent versus applied bias voltage, shown as black triangles (smooth-textured InP), purple diamonds (fine-textured $\mathrm{InP}$ ), and green squares (coarse-textured InP). It is clear that there is a very large photocurrent in the PC $\mathrm{THz}$ emitter that is smooth-textured. One the other hand, there is a medium photocurrent in the PC THz emitter that is fine-textured and a low photocurrent in the PC $\mathrm{THz}$ emitter that is coarse-textured. Of particular interest is the ratio between the $\mathrm{THz}$ normalized electric field amplitude divided by the photocurrent, $E_{\mathrm{THz}, \mathrm{norm}}\left(V_{\text {bias }}\right) / I_{\mathrm{ph}}\left(V_{\text {bias }}\right)$. This quantifies Joule heating

(a) smooth-textured InP

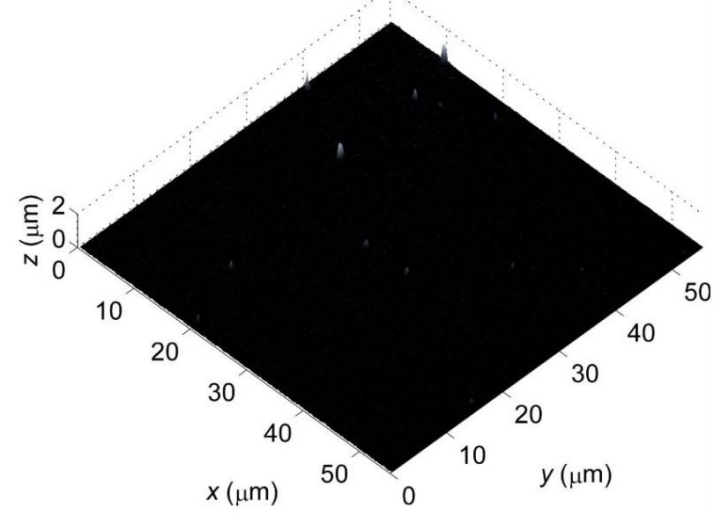

(b) fine-textured InP

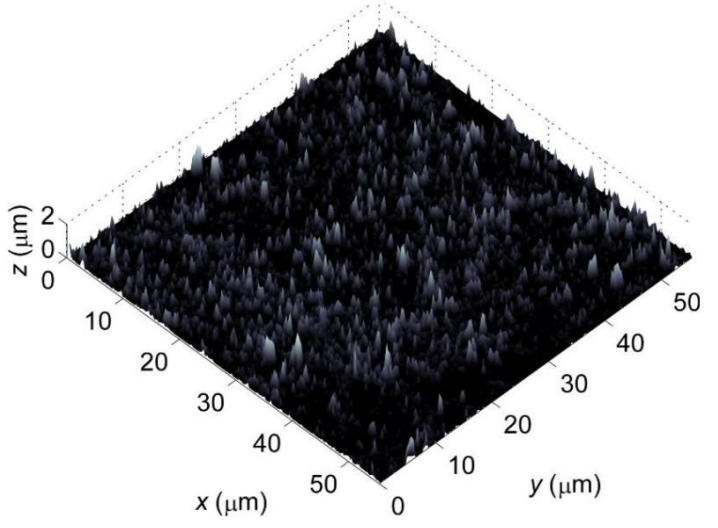

(c) coarse-textured InP

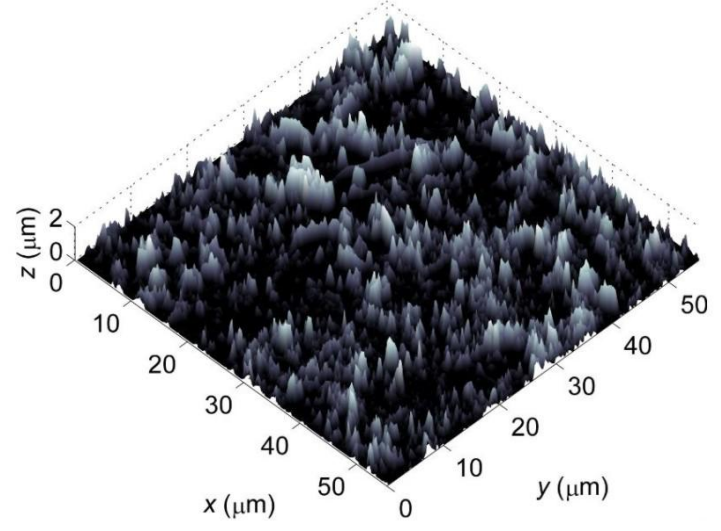

Figure 2. Topographies based on SEM images for (a) smooth-, (b) fine-, and (c) coarse-textured InP materials. 
and ohmic losses. Figure 4(c) presents this ratio versus applied voltage, $V_{\text {bias }}$, shown as black triangles (smooth-textured InP), purple diamonds (fine-textured InP), and green squares (coarse-textured InP). The ratio measures are taken at six $\mathrm{PC} \mathrm{THz}$ emitter locations and the mean is presented in Figure 4(c). Error bars indicate the standard error of the measurements. The conversion from photocurrent to $\mathrm{THz}$ electric field is much better for textured $\mathrm{PC} \mathrm{THz}$ emitters.

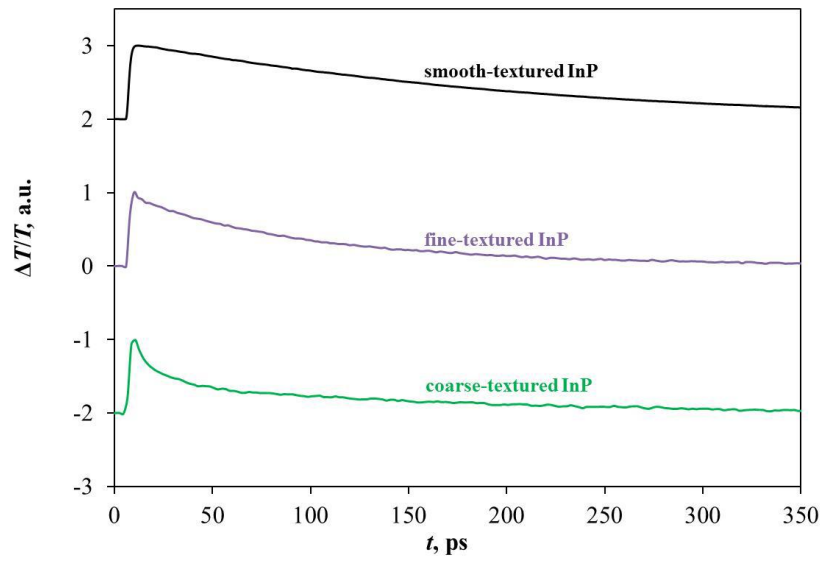

Figure 3. Differential transmission, $\Delta T / T$, as a function of time, $t$, for pump-probe scans of smooth-, fine-, and coarse-textured InP materials. The scans are used to identify the charge-carrier lifetimes for the three materials.

(a)

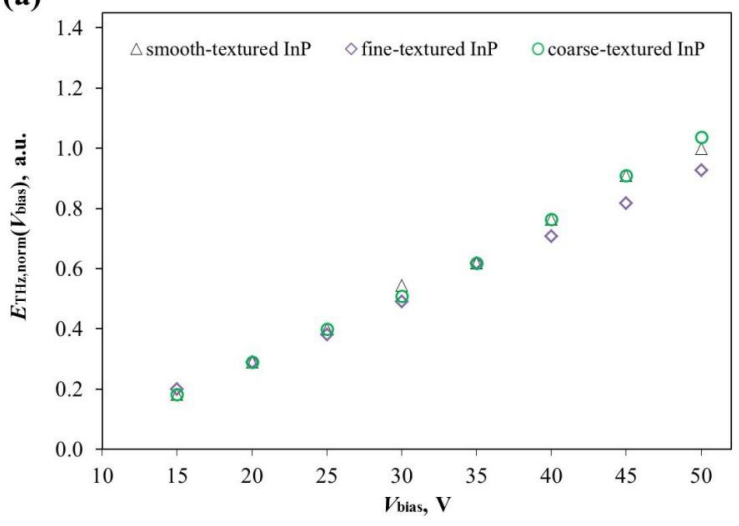

(b)

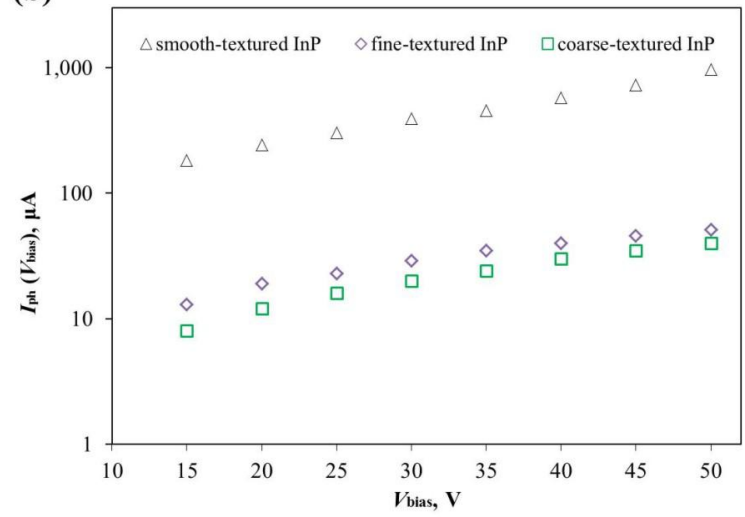

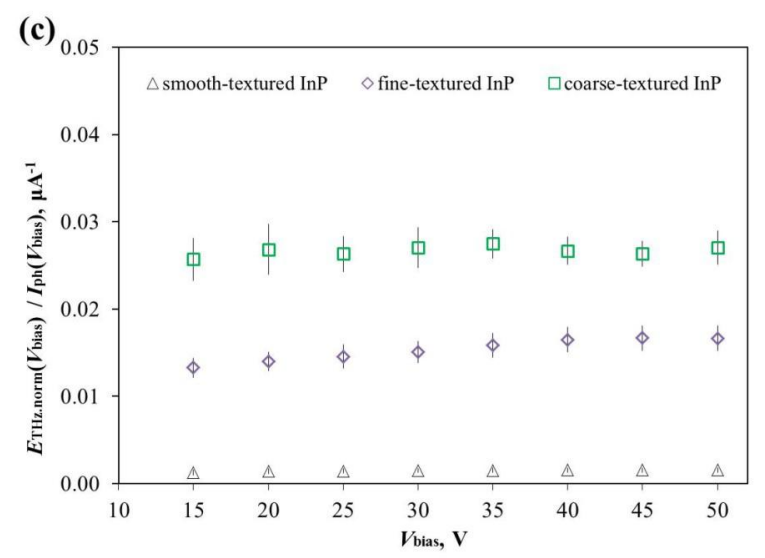

Figure 4. Shown are the THz responses for the InP PC THz emitters that are smooth-, fine-, and coarse-textured with (a) THz electric field versus applied bias voltage, (b) photocurrent versus applied bias voltage, and (c) normalized $\mathrm{THz}$ electric field divided by photocurrent versus applied bias voltage. 
(a)

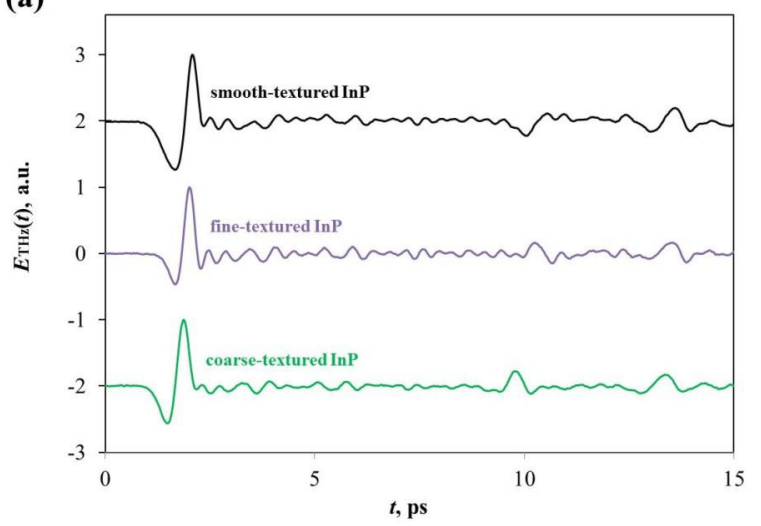

(b)

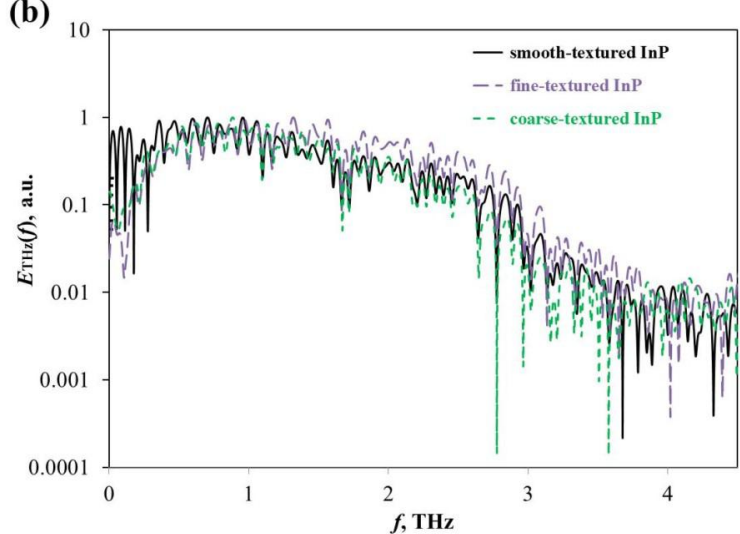

Figure 5. The (a) time- and (b) frequency-domain THz responses of the PC THz emitters using smooth-, fine-, and coarse-textured InP materials.

The time- and frequency-domain response of the PC THz emitters can also be tested, as shown in Figure 5. Figure 5(a) and 5(b) show the time-domain response and frequency-domain $\mathrm{THz}$ responses of each emitter. The scans are normalized to its amplitude. The three emitters all have a similar, $3 \mathrm{THz}$, bandwidth. Thus, it can be concluded that the residual photocurrent can be reduced, lowering ohmic losses and Joule heating, without losing bandwidth through texturing semiconductors in $\mathrm{PC} \mathrm{THz}$ emitters.

\section{CONCLUSIONS}

This work investigated PC THz emitters constructed with textured InP materials. These emitters lowered ohmic losses and Joule heating due to their lowered charge-carrier lifetimes and were found to consume much less photocurrent. This material texturing is useful to create $\mathrm{PC} \mathrm{THz} \mathrm{emitters} \mathrm{that} \mathrm{can} \mathrm{mitigate} \mathrm{premature} \mathrm{device} \mathrm{breakdown} \mathrm{through} \mathrm{thermal}$ runaway. Such emitters could be used in small-scale and/or high-power THz applications.

\section{ACKNOWLEDGMENTS}

This work was supported by the Natural Sciences and Engineering Research Council of Canada (NSERC).

\section{REFERENCES}

[1] O’Callahan, B. T. et al., "Inhomogeneity of the ultrafast insulator-to-metal transition dynamics of VO2," Nature Communications 6, 6849 (2015).

[2] Jin, X. et al., "Ultrafast transient responses of optical wireless communication detectors," Applied Optics 52, 50425049 (2013).

[3] Hoy, C. L. et al., "Clinical ultrafast laser surgery: Recent advances and future directions," IEEE Journal of Selected Topics in Quantum Electronics 20, 7100814 (2014).

[4] Castro-Camus, E., Lloyd-Hughes, J., and Johnston, M. B., "Three-dimensional carrier-dynamics simulation of terahertz emission from photoconductive switches," Physical Review B 71, 195301 (2005).

[5] Chen, S.-L. et al., "Efficient real-time detection of terahertz pulse radiation based on photoacoustic conversion by carbon nanotube nanocomposite," Nature Photonics 8, 537-542 (2014).

[6] Huber, R., "Photonics: Terahertz collisions," Nature 483, 545-546 (2012).

[7] Collier, C. M. et al., "Optimization processes for pulsed terahertz systems," Applied Optics 54, 535-545 (2015). 
[8] Shalaby, M. and Hauri, C. P., "Spectrally intense terahertz source based on triangular Selenium," Scientific Reports 5, 8059 (2015).

[9] Pavlov, S. G. et al., "Terahertz stimulated emission from silicon doped by hydrogenlike acceptors," Physical Review X 4, 021009 (2014).

[10] Jewariya, M. et al., "Fast three-dimensional terahertz computed tomography using real-time line projection of intense terahertz pulse," Optics Express 21, 2423-2433 (2013).

[11] Shinokita, K. et al., "Terahertz-induced optical emission of photoexcited undoped GaAs quantum wells," Physical Review Letters 111, 067401 (2013).

[12] Teich, M. et al., "Systematic investigation of terahertz-induced excitonic Rabi splitting," Physical Review B 89, 115311 (2014).

[13] Ho, L., Pepper, M., and Taday, P., "Terahertz technology seems set to become important in security screening and the pharmaceutical industry," Nature Photonics 2, 541-543 (2008).

[14] Tonouchi, M. "Cutting-edge terahertz technology," Nature Photonics 1, 97-105 (2007).

[15] Nakajima, S. et al., "Terahertz imaging diagnostics of cancer tissues with a chemometrics technique," Applied Physics Letters 90, 041102 (2007).

[16] Wu, X., Yiwen, E., Xu, X., and Wang, L., "Label-free monitoring of interaction between DNA and oxaliplatin in aqueous solution by terahertz spectroscopy," Applied Physics Letters 101, 033704 (2012).

[17] Marandi, A., Darcie, T. E., and So, P. P. M., "Design of a continuous-wave tunable terahertz source using waveguide-phase-matched GaAs," Optics Express 16, 10427-10433 (2008).

[18] Preu, S. et al., "Tunable, continuous-wave Terahertz photomixer sources and applications," Journal of Applied Physics 109, 061301 (2011).

[19] Tomasino, A. et al., "Wideband THz time domain spectroscopy based on optical rectification and electro-optic sampling," Scientific Reports 3, 3116 (2013).

[20]Beck, M. et al., "Impulsive terahertz radiation with high electric fields from an amplifier-driven large-area photoconductive antenna," Optics Express 18, 9251-9257 (2010).

[21] Singh, A. et al., "Highly efficient and electrically robust carbon irradiated semi-insulating GaAs based photoconductive terahertz emitters," Applied Physics Letters 104, 063501 (2014).

[22] Stone, M. R., Naftaly, M., Miles, R. E., Fletcher, J. R. and Steenson, D. P., "Electrical and radiation characteristics of semilarge photoconductive terahertz emitters," IEEE Transactions on Microwave Theory and Techniques 52, 2420-2429 (2004).

[23]F. Buccheri and X.-C. Zhang, "Terahertz emission from laser-induced microplasma in ambient air," Optica 2, 366369 (2015).

[24] Mittendorff, M. et al., "Large area photoconductive terahertz emitter for $1.55 \mu \mathrm{m}$ excitation based on an InGaAs heterostructure," Nanotechnology 24, 214007 (2013).

[25] George, P. A. et al., "Microfluidic devices for terahertz spectroscopy of biomolecules," Optics Express 16, 1577 $1582(2008)$.

[26] Nichols, J. et al., "On-chip digital microfluidic architectures for enhanced actuation and sensing," Journal of Biomedical Optics 17, 067005 (2012).

[27] Collier, C. M. et al., "Nonlinear dual-phase multiplexing in digital microfluidic architectures," Micromachines 2, 369-384 (2011).

[28] Planken, P. C. M., van Rijmenam, C. E. W. M., and Schouten, R. N., "Opto-electronic pulsed THz systems," Semiconductor Science and Technology 20, S121-S127 (2005).

[29] Berry, C. W. et al., "Significant performance enhancement in photoconductive terahertz optoelectronics by incorporating plasmonic contact electrodes," Nature Communications 4, 1622 (2013).

[30] Park, S.-G. et al., "Enhancement of terahertz pulse emission by optical nanoantenna," ACS Nano 6, 2026-2031 (2012).

[31] Ackemann, T. et al., "Diamond heat sinking of terahertz antennas for continuous-wave photomixing," Journal of Applied Physics 112, 123109 (2012).

[32] Collier, C. M. and Holzman, J. F. "Ultrafast photoconductivity of crystalline, polycrystalline, and nanocomposite ZnSe material systems for terahertz applications," Applied Physics Letters 104, 042101 (2014).

[33] Collier, C. M., Born, B. and Holzman, J. F., "Ultrafast response of SiC and Si nanocomposite material systems," Electronics Letters 48, 1618-1619 (2012).

[34] Collier, C. M., Jin, X., and Holzman, J. F., "Ultrafast refractometry for characterization of nanocomposite material systems," IEEE Photonics Technology Letters 24, 590-592 (2012). 
[35] Reid, M. and Fedosejevs, R. "Quantitative comparison of terahertz emission from (100) InAs surfaces and a GaAs large-aperture photoconductive switch at high fluences," Applied Optics 44, 149-153 (2005).

[36] Collier, C. M. et al., "Ultrafast photoexcitation and transient mobility of GaP for PC terahertz emission," IEEE Journal of Quantum Electronics 49, 691-696 (2013).

[37] Collier, C. M., Born, B., Jin, X., and Holzman, J. F., "Ultrafast charge-carrier and phonon dynamics in GaP," Applied Physics Letters 103, 072106 (2013).

[38] Waldmüller, S., Lang, M., Wellmann, P. and Winnacker, A., "Spatial distribution of charge carrier temperature and lifetime in semi-insulating InP:Fe, observed via photoluminescence spectroscopy," Proceedings of the Sixth International Conference on Indium Phosphide and Related Materials 18, 213 (1994). 